\title{
An easily calculable and highly predictive risk index for postoperative renal failure after heart transplantation
}

\author{
Arman Kilic, MD, Joshua C. Grimm, MD, Ashish S. Shah, MD, John V. Conte, MD, \\ Glenn J. R. Whitman, MD, and Christopher M. Sciortino, MD, PhD
}

\begin{abstract}
Objectives: This study derived and validated a risk index for postoperative renal failure after orthotopic heart transplantation.

Methods: Adult orthotopic heart transplantations performed between 2000 and 2010 were identified in the United Network for Organ Sharing database. Patients were randomly divided 4:1 into derivation and validation cohorts. The primary outcome was new-onset postoperative renal failure requiring dialysis. A multivariable model was created incorporating variables associated with renal failure in univariate analysis, with significant risk factors assigned points based on odds ratios. A simple guide was generated to demonstrate ranges of risk scores associated with clinically meaningful renal failure rates.
\end{abstract}

Results: A total of 14,635 orthotopic heart transplantation recipients were included. New-onset postoperative renal failure occurred in 1128 patients $(7.7 \%)$. A 100-point risk score was generated using 13 significant risk factors. There was a high degree of correlation between actual renal failure rates in the validation cohort and predicted rates in the derivation cohort based on risk scores $(r=0.91, P<.001)$. Renal failure risk categories were generated on the basis of probabilities determined in the derivation cohort: low $(<5 \%$ risk, score $0-15)$, average (5\%-10\% risk, score $16-26)$, above average (10\%-20\% risk, score $27-39)$, and high ( $>20 \%$ risk, score $\geq 40)$. The actual renal failure rates in the validation cohort for these risk score ranges corresponded to the risk category they were assigned to: score 0 to 15 ( $4.1 \%$ rate), score 16 to 26 (6.8\% rate), score 27 to 39 (13.2\% rate), and score 40 or more $(20.2 \%$ rate)

Conclusions: This 100-point risk index incorporating 13 risk factors is highly predictive of new-onset postoperative renal failure after orthotopic heart transplantation. Prospective assessment of orthotopic heart transplant recipients using the risk categories that were generated on the basis of score ranges may help in tailoring perioperative management. (J Thorac Cardiovasc Surg 2014;148:1099-105)

\begin{abstract}
Although variable, the incidence of acute kidney injury after orthotopic heart transplantation (OHT) has been reported as high as $40 \%$ to $70 \%{ }^{1-3}$ Acute kidney failure represents a substantial proportion of these cases and is associated with postoperative mortality rates of upward of $35 \%$ to $50 \%$. $^{2,4}$ Furthermore, postoperative acute renal failure represents a significant risk factor for developing chronic kidney disease after OHT, which occurs in approximately $10 \%$ of OHT recipients by 5 years post-transplant. ${ }^{5}$ Beyond its direct impact on patient outcomes, acute renal failure is associated with significant increases in resource use and cost. ${ }^{6}$

Although risk factors for acute renal failure after OHT have been identified, there is no simple clinical guide to estimate

\footnotetext{
From the Division of Cardiac Surgery, Department of Surgery, The Johns Hopkins Medical Institutions, Baltimore, Md.

Disclosures: Authors have nothing to disclose with regard to commercial support.

Read at the 94th Annual Meeting of The American Association for Thoracic

Surgery, Toronto, Ontario, Canada, April 26-30, 2014.

Received for publication April 6, 2014; revisions received May 27, 2014; accepted for publication May 30, 2014; available ahead of print July 1, 2014.

Address for reprints: Christopher M. Sciortino, MD, PhD, Division of Cardiac Surgery, Johns Hopkins Hospital, 1800 Orleans St, Sheikh Zayed Tower Suite 7107, Baltimore, MD 21287 (E-mail: csciort2@jhmi.edu). $0022-5223 / \$ 36.00$

Copyright (C) 2014 by The American Association for Thoracic Surgery
} http://dx.doi.org/10.1016/j.jtcvs.2014.05.065
\end{abstract}

overall risk. Such a guide would be useful to clinicians in tailoring perioperative management and stratifying patients for research studies according to baseline risk. The objective of this study was to derive and validate a composite risk index for postoperative renal failure after OHT.

\section{METHODS \\ Database \\ This study used the United Network for Organ Sharing (UNOS) database. This registry contains patient-level data on all solid-organ transplants performed in the United States. The institutional review board granted this study exempt status because the data are publically available and de-identified. All statistical analyses were performed using Stata version 11 software (StataCorp LP, College Station, Tex).}

\section{Study Population}

The study population consisted of adult patients aged 18 years or more undergoing OHT in the United States between January 1, 2000, and December 31, 2010. Patients undergoing simultaneous multiorgan transplants or redo OHT were excluded from analysis. Patients on dialysis before OHT also were excluded.

\section{Baseline Study Population Characteristics}

Baseline characteristics were initially summarized. These included recipient, donor, and operative variables. All variables provided by 


\section{Abbreviations and Acronyms \\ $\mathrm{CI}=$ confidence interval \\ OHT $=$ orthotopic heart transplantation \\ UNOS $=$ United Network for Organ Sharing}

UNOS were evaluated. The full listing of variables available in the UNOS registry can be found at http://www.unos.org.

\section{Derivation and Validation Cohorts}

The study population was randomly divided into derivation and validation cohorts in a $4: 1$ ratio. Baseline characteristics were then compared between the derivation and validation cohorts. These included all recipient, donor, and operative variables.

\section{Generation of Risk Index in the Derivation Cohort}

The primary outcome for this analysis was new-onset postoperative acute renal failure requiring dialysis. The association between each variable provided by UNOS and postoperative acute renal failure was tested in univariate logistic regression analysis in the derivation cohort. Variables associated with postoperative renal failure in univariate analysis (exploratory $P$ value $<.20$ ) were then entered into a multivariable logistic regression model. Variables with $>25 \%$ missing data were excluded from entry into the model. The inclusion of covariates and performance of the final multivariable model were evaluated using the Hosmer-Lemeshow goodness-of-fit test, McFadden's pseudo- $\mathrm{R}^{2}$, Akaike information criterion, likelihood ratio test, and area under the receiver operating curve. Continuous data were categorized on the basis of receiver operating characteristic analysis and graphically displaying splines to identify break points. Points were then assigned to each significant covariate in the final multivariable model. The magnitude of the points corresponded to the relative magnitude of the odds ratio for that covariate. Moreover, we multiplied the odds ratio by a factor of 4 and rounded to the nearest whole number to arrive at the number of risk points assigned to each risk factor. A composite risk index was then generated for each patient by simply summing the individual points. A probability curve was generated to visually display the odds of postoperative renal failure for each score based on the derivation cohort.

\section{Validation of Risk Index}

Composite risk scores were calculated for each patient in the validation cohort. The performance of the risk index in the validation cohort was initially evaluated using logistic regression analysis. A weighted regression analysis was performed to evaluate the association between predicted renal failure rates based on the derivation cohort for each score and actual observed renal failure rates in the validation cohort for that same score. Weights were assigned on the basis of the frequency of each score.

\section{Clinical Guide to Estimate Renal Failure Risk}

A simple guide was generated that demonstrated the range of risk scores associated with clinically meaningful renal failure rates in the derivation cohort. The categories of renal failure risk were defined as follows: low $(<5 \%)$, average $(5 \%-10 \%)$, above average $(10 \%-20 \%)$, and high $(\geq 20 \%)$. The average renal failure rate observed in the validation cohort for patients within these same ranges of risk scores were then evaluated to determine whether they corresponded to the rates provided in the guide.

\section{RESULTS}

\section{Baseline Characteristics}

A total of 14,635 patients were included in this analysis. The average recipient age was $52.2 \pm 12.2$ years (Table 1 ).
Some $76 \%$ of patients were male, and $73 \%$ were Caucasian. There was a similar proportion of ischemic heart disease and idiopathic dilated cardiomyopathy. The mean preoperative serum creatinine was $1.27 \pm 0.46 \mathrm{mg} / \mathrm{dL}$. Only $2 \%$ and $5 \%$ of patients required mechanical ventilation or an intra-aortic balloon pump before OHT, respectively, although $21 \%$ were bridged with a ventricular assist device. In comparing the derivation and validation cohorts, there were no differences in key recipient characteristics except for serum bilirubin, which was higher in the validation cohort (Table 1).

The mean donor age was $31.4 \pm 12.2$ years (Table 1 ). Similar to recipients, most donors were male and Caucasian. In $59 \%$ of donors, the mechanism of death was trauma-related. The average center OHT volume was 24.7 \pm 17.7 per year. The mean ischemic time was $3.20 \pm$ 1.04 hours. There were no differences in donor or operative characteristics between the derivation and validation cohorts (Table 1).

\section{Generation of Risk Score in the Derivation Cohort}

New-onset postoperative renal failure occurred in 1128 patients $(7.7 \%)$ in the entire study population. A total of 11,689 patients $(80 \%)$ were randomly assigned to the derivation cohort. A total of 15 variables were associated with postoperative renal failure in the derivation cohort in univariate analysis and therefore included in the multivariable model: older age, congenital heart disease or "other" heart failure cause, reduced creatinine clearance, increased serum bilirubin, increased body mass index, diabetes mellitus, mechanical ventilation, intensive care unit before OHT, recent infection requiring intravenous antibiotics, blood transfusion while on the waitlist, previous malignancy, older donor age, female donor, biatrial anastomosis, and longer ischemic time (Table 2). The risk factors that were significant predictors of renal failure in the final model included age 60 years or more, congenital heart disease or "other" heart failure cause, creatinine clearance less than $60 \mathrm{~mL} / \mathrm{min}$, serum bilirubin $1 \mathrm{mg} / \mathrm{dL}$ or greater, body mass index $30 \mathrm{~kg} / \mathrm{m}^{2}$ or greater, diabetes mellitus, mechanical ventilation, intensive care unit before OHT, recent infection, blood transfusion on waitlist, donor age 30 years or more, biatrial anastomosis, and ischemic time 4 hours or more (Table 2). The final multivariable model had an area under receiver operating characteristic curve of 0.70 , Akaike information criterion of 5896, and a nonsignificant Hosmer-Lemeshow chisquare of 15.4. Individual risk points varied from as low as 5 each for age 60 years or more, serum bilirubin 1 to 1.9 $\mathrm{mg} / \mathrm{dL}$, body mass index 30 to $34.9 \mathrm{~kg} / \mathrm{m}^{2}$, diabetes mellitus, intensive care unit before OHT, and donor age 30 to 49 years, to 15 points for creatinine clearance less than $30 \mathrm{~mL} / \mathrm{min}$ and 16 points for congenital heart disease. Overall, the composite risk index was out of 100 possible points. 
TABLE 1. Baseline study population characteristics

\begin{tabular}{|c|c|c|c|c|}
\hline Variable & Study population $(n=14,635)$ & Derivation cohort $(n=11,689)$ & Validation cohort $(n=2946)$ & $P$ value* \\
\hline \multicolumn{5}{|l|}{ Recipient } \\
\hline Age (y) & $52.2 \pm 12.2$ & $52.1 \pm 12.2$ & $52.5 \pm 12.2$ & .21 \\
\hline Female & $3495(24 \%)$ & $2757(24 \%)$ & $738(25 \%)$ & .10 \\
\hline Caucasian race & $10,689(73 \%)$ & $8519(73 \%)$ & $2170(74 \%)$ & .50 \\
\hline Heart failure cause & & & & .98 \\
\hline Ischemic & $6477(44 \%)$ & $5163(44 \%)$ & $1314(45 \%)$ & \\
\hline Dilated CM & $6316(43 \%)$ & $5054(43 \%)$ & $1262(43 \%)$ & \\
\hline Congenital & $369(3 \%)$ & $294(3 \%)$ & $75(3 \%)$ & \\
\hline Other & $1473(10 \%)$ & $1178(10 \%)$ & $295(10 \%)$ & \\
\hline Diabetes mellitus & $3398(23 \%)$ & $2692(23 \%)$ & $706(24 \%)$ & .28 \\
\hline Body mass index $\left(\mathrm{kg} / \mathrm{m}^{2}\right)$ & $26.7 \pm 4.9$ & $26.7 \pm 4.9$ & $26.6 \pm 4.9$ & .22 \\
\hline Serum creatinine $(\mathrm{mg} / \mathrm{dL})$ & $1.27 \pm 0.46$ & $1.26 \pm 0.46$ & $1.27 \pm 0.47$ & .38 \\
\hline Serum bilirubin (mg/dL) & $1.22 \pm 1.98$ & $1.21 \pm 1.85$ & $1.29 \pm 2.46$ & .03 \\
\hline Mechanical ventilation & $329(2 \%)$ & $258(2 \%)$ & $71(2 \%)$ & .51 \\
\hline Intra-aortic balloon pump & $704(5 \%)$ & $561(5 \%)$ & $143(5 \%)$ & .90 \\
\hline Bridged with ventricular assist device & $3076(21 \%)$ & $2460(21 \%)$ & $616(21 \%)$ & .87 \\
\hline Intensive care unit & $4203(29 \%)$ & $3350(29 \%)$ & $853(29 \%)$ & .75 \\
\hline Recent infection & $1517(10 \%)$ & $1209(10 \%)$ & $308(10 \%)$ & .86 \\
\hline Blood transfusion on waitlist & $2993(20 \%)$ & $2377(20 \%)$ & $616(21 \%)$ & .49 \\
\hline \multicolumn{5}{|l|}{ Donor } \\
\hline Age (y) & $31.4 \pm 12.2$ & $31.4 \pm 12.2$ & $31.4 \pm 12.2$ & .84 \\
\hline Female & $4092(28 \%)$ & $3264(28 \%)$ & $828(28 \%)$ & .84 \\
\hline Caucasian race & $9966(68 \%)$ & $7986(68 \%)$ & $1980(67 \%)$ & .25 \\
\hline Mechanism of death & & & & .73 \\
\hline Trauma & $8609(59 \%)$ & $6858(59 \%)$ & $1751(59 \%)$ & \\
\hline Cerebrovascular & $4656(32 \%)$ & $3736(32 \%)$ & $920(31 \%)$ & \\
\hline Other & $1366(9 \%)$ & $1092(9 \%)$ & $274(9 \%)$ & \\
\hline \multicolumn{5}{|l|}{ Operative } \\
\hline Biatrial anastomosis & $8619(59 \%)$ & $6884(59 \%)$ & $1735(59 \%)$ & .99 \\
\hline Average annual center OHT volume & $24.7 \pm 17.7$ & $24.6 \pm 17.6$ & $24.9 \pm 17.9$ & .50 \\
\hline Ischemic time $(\mathrm{h})$ & $3.20 \pm 1.04$ & $3.20 \pm 1.04$ & $3.20 \pm 1.05$ & .93 \\
\hline
\end{tabular}

The mean composite score in the derivation cohort was $19.8 \pm 9.8$, with a range of 0 to 76 . A probability curve of predicted postoperative renal failure rates based on risk score was generated using derivation cohort data (Figure 1). The predicted renal failure rate ranged from $1.8 \%(95 \%$ confidence interval $[\mathrm{CI}], 1.5-2.2)$ for a score of 0 to $74.0 \%$ (95\% CI, 66.8-80.0) for a score of 76 . A simple guide was constructed that provided a range of risk scores associated with clinically meaningful renal failure rates (Table 3).

\section{Validation of Risk Score in the Validation Cohort}

A total of 2946 patients $(20 \%)$ were randomly assigned to the validation cohort. The mean risk score in the validation cohort was $20.2 \pm 9.8$, with a range of 0 to 57 . The risk score was highly predictive of postoperative renal failure requiring dialysis in the validation cohort (odds ratio, 1.06; 95\% CI, 1.04-1.07; $P<.001)$. The multivariable model had similar performance in the validation cohort as the derivation cohort with a c-index of 0.70, Akaike information criterion of 1526, and nonsignificant Hosmer-Lemeshow chi-square of 3.7. In weighted regression analysis, there was a high degree of correlation between predicted renal failure rates based on the derivation cohort and actual renal failure rates in the validation cohort $(r=0.91, P<.001)$ (Figure 2).

The actual renal failure rates in the validation cohort for the ranges of scores in the guide in Table 3 were within the range of predicted rates based on the derivation cohort (Figure 3). Moreover, in the validation cohort, patients predicted to be low risk with a score of 0 to 15 had an actual renal failure rate of $4.1 \%$. Patients predicted to be average risk with a score of 16 to 26 had an actual renal failure rate of $6.8 \%$. Likewise, patients predicted to be at above average risk with a score of 27 to 39 had an actual renal failure rate of $13.2 \%$. Finally, patients in the validation cohort predicted to be at high risk based on the derivation cohort with a score of 40 or higher had an actual renal failure rate of $20.2 \%$. These rates corresponded well to the renal failure rates observed in the derivation cohort for the 
TABLE 2. Final multivariable logistic regression model used to construct risk score for new-onset postoperative acute renal failure requiring dialysis

\begin{tabular}{|c|c|c|c|}
\hline Covariates & $\begin{array}{c}\text { Odds ratio } \\
(95 \% \mathrm{CI})\end{array}$ & $P$ value & $\begin{array}{c}\text { Points } \\
\text { assigned }\end{array}$ \\
\hline \multicolumn{4}{|l|}{ Recipient } \\
\hline Age $\geq 60 y$ & $1.21(1.03-1.42)$ & .02 & 5 \\
\hline \multicolumn{4}{|l|}{ Heart failure cause } \\
\hline Dilated CM & Reference & Ref. & 0 \\
\hline Ischemic heart disease & $0.98(0.83-1.15)$ & .81 & 0 \\
\hline Congenital heart disease & $4.01(2.88-5.60)$ & $<.001$ & 16 \\
\hline Other & $1.54(1.22-1.93)$ & $<.001$ & 6 \\
\hline \multicolumn{4}{|l|}{$\begin{array}{l}\text { Creatinine clearance } \\
\qquad(\mathrm{mL} / \mathrm{min})\end{array}$} \\
\hline$\geq 60$ & Reference & Ref. & 0 \\
\hline $30-59$ & $1.92(1.65-2.23)$ & $<.001$ & 8 \\
\hline$<30$ & $3.86(2.80-5.32)$ & $<.001$ & 15 \\
\hline \multicolumn{4}{|l|}{ Serum bilirubin $(\mathrm{mg} / \mathrm{dL})$} \\
\hline$<1$ & Reference & Ref. & 0 \\
\hline $1-1.9$ & $1.29(1.10-1.51)$ & .002 & 5 \\
\hline $2-2.9$ & $1.55(1.19-2.04)$ & .001 & 6 \\
\hline$\geq 3$ & $1.78(1.38-2.30)$ & $<.001$ & 7 \\
\hline \multicolumn{4}{|l|}{ Body mass index $\left(\mathrm{kg} / \mathrm{m}^{2}\right)$} \\
\hline$<18.5$ & Reference & Ref. & 0 \\
\hline $18.5-24.9$ & $1.24(0.84-1.82)$ & .29 & 0 \\
\hline $25-29.9$ & $1.14(0.96-1.35)$ & .14 & 0 \\
\hline $30-34.9$ & $1.36(1.11-1.67)$ & .003 & 5 \\
\hline$\geq 35$ & $1.43(1.03-1.99)$ & .04 & 6 \\
\hline Diabetes mellitus & $1.26(1.07-1.49)$ & .006 & 5 \\
\hline Mechanical ventilation & $2.31(1.66-3.23)$ & $<.001$ & 9 \\
\hline Intensive care unit & $1.32(1.13-1.54)$ & $<.001$ & 5 \\
\hline Recent infection & $1.59(1.30-1.95)$ & $<.001$ & 6 \\
\hline $\begin{array}{l}\text { Blood transfusion on } \\
\text { waitlist }\end{array}$ & $1.43(1.21-1.69)$ & $<.001$ & 6 \\
\hline Previous malignancy & $0.93(0.68-1.27)$ & .65 & Not included \\
\hline \multicolumn{4}{|l|}{ Donor } \\
\hline \multicolumn{4}{|l|}{ Donor age (y) } \\
\hline$<30$ & Reference & Ref. & 0 \\
\hline $30-49$ & $1.19(1.02-1.39)$ & .02 & 5 \\
\hline$\geq 50$ & $1.69(1.35-2.12)$ & $<.001$ & 7 \\
\hline Female donor & $1.09(0.93-1.28)$ & .27 & Not included \\
\hline \multicolumn{4}{|l|}{ Operative } \\
\hline Biatrial anastomosis & $1.19(1.04-1.38)$ & .01 & 5 \\
\hline \multicolumn{4}{|l|}{ Ischemic time (h) } \\
\hline$<4$ & Reference & Ref. & 0 \\
\hline $4-5.9$ & $1.43(1.21-1.68)$ & $<.001$ & 6 \\
\hline$\geq 6$ & $1.92(1.15-3.22)$ & .01 & 8 \\
\hline
\end{tabular}

$C I$, Confidence interval; $C M$, cardiomyopathy.

same range of risk scores: score 0 to $15(3.3 \%)$, score 16 to $26(7.1 \%)$, score 27 to $39(13.7 \%)$, and score 40 or more $(23.9 \%)$ (Figure 3$)$. There was also a correlation between risk score and 30-day mortality, with 30-day mortality rates that were $2.1 \%, 4.2 \%, 7.4 \%$, and $15.8 \%$ for patients with scores associated with the lowest to highest renal failure risk categories, respectively (odds ratio for 30-day mortality, $1.06 ; 95 \% \mathrm{CI}, 1.06-1.07 ; P<.001)$.

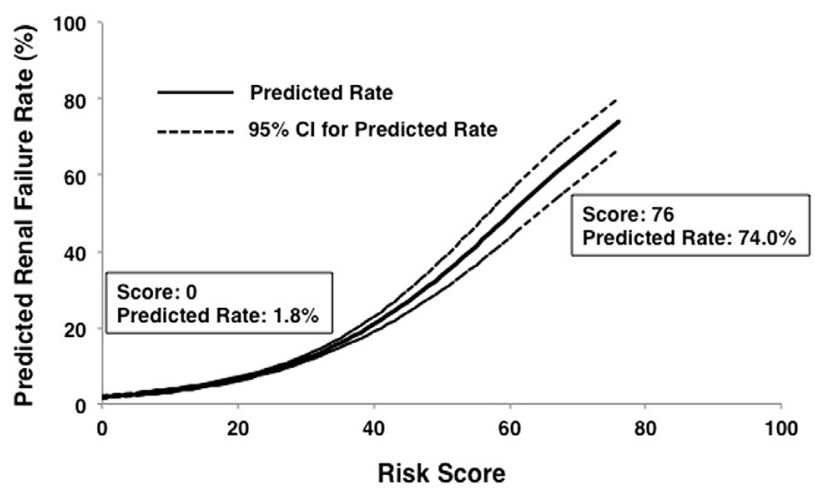

FIGURE 1. Probability of new-onset postoperative acute renal failure based on risk score in the derivation cohort. $C I$, Confidence interval.

\section{DISCUSSION}

In this study of 14,635 patients undergoing OHT, we derived and validated a 100-point risk score for new-onset postoperative acute renal failure requiring dialysis incorporating 13 recipient, donor, and operative variables.

\section{Individual Risk Factors for Renal Failure}

The strongest risk factors for postoperative renal failure in our analysis included congenital heart disease, reduced preoperative creatinine clearance, mechanical ventilation before OHT, and prolonged ischemic time. In a prior UNOS analysis of 689 adult patients with congenital heart disease undergoing OHT, the rate of postoperative dialysis was $18 \%$ in this higher-risk patient subset compared with $8 \%$ in OHT recipients with other heart failure causes. ${ }^{7}$ Another study of 756 OHT recipients between 1993 and 2004 found that increased preoperative serum creatinine was a significant risk factor for postoperative acute kidney injury in multivariable analysis. ${ }^{4}$ Other significant predictors in the latter study included lower serum albumin level, insulin-dependent diabetes, and longer cardiopulmonary bypass time.

In a pooled analysis of thoracic organ transplants at a single center, mechanical ventilation was found to correlate with the degree of acute kidney injury, with intraoperative blood transfusion and preexisting impaired renal function being independent predictors. ${ }^{8}$ Prolonged ischemic time also has been demonstrated to be a risk factor for acute renal failure after OHT. $^{9}$

TABLE 3. A simple guide demonstrating the range of risk scores associated with clinically meaningful new-onset postoperative acute renal failure rates

\begin{tabular}{lcl}
\hline Range of risk scores & $\begin{array}{c}\text { Predicted postoperative } \\
\text { renal failure rate }\end{array}$ & Risk category \\
\hline $0-15$ & $<5 \%$ & Low \\
$16-26$ & $5 \%-10 \%$ & Average \\
$27-39$ & $10 \%-20 \%$ & Above average \\
$\geq 40$ & $\geq 20 \%$ & High \\
\hline
\end{tabular}




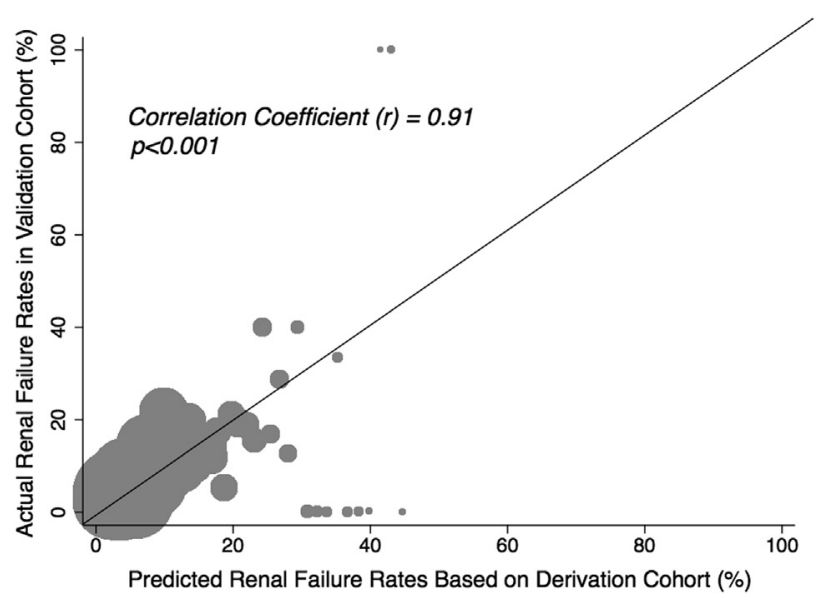

FIGURE 2. Weighted regression analysis demonstrating the significant association between predicted renal failure rates based on the derivation cohort and actual observed renal failure rates in the validation cohort.

An analysis of $65 \mathrm{OHT}$ recipients identified recipient age, which was included in our risk score as a significant risk factor, and the use of cyclosporine as independent predictors of postoperative renal failure. ${ }^{3}$ Of note, biatrial anastomosis was identified as a risk factor in our study. Prior studies have demonstrated decreased survival with the biatrial technique in part due to worse hemodynamics and right ventricular function compared with the bicaval technique. $^{10,11}$ Although not directly reported, it is conceivable that the higher likelihood of impaired forward flow with the biatrial technique would predispose OHT recipients to an increased risk of renal failure when compared with bicaval anastomoses.

\section{Clinical Utility of the Risk Score}

A risk score to be used in the clinical realm ideally should be easy to calculate. Some risk scores, such as the Society for Thoracic Surgeons' risk calculators for coronary artery bypass grafting or valve surgery, are able to simplify the process through web-based entry and automated calculation. ${ }^{12,13}$ We believe our risk score fulfills this criteria because the components of the score are easy to

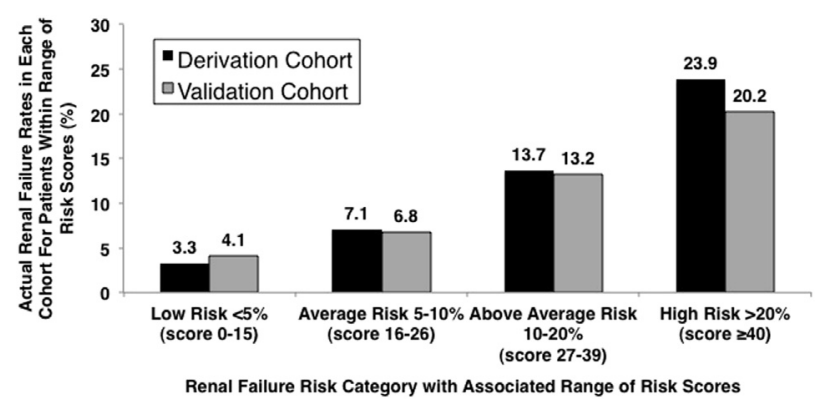

FIGURE 3. Actual rates of new-onset postoperative acute renal failure by risk categories. measure and whole points are assigned to the individual risk factors.

Another important aspect of a clinically useful risk score is that a wide range of outcomes are predicted when comparing scores at the low versus high end of the risk spectrum. Again, we believe our composite risk index fulfills this criteria. In the derivation cohort, the predicted risk of renal failure ranged from a very low $1.8 \%$ for a score of 0 to a very high risk of $74.0 \%$ for a score of 76 , clearly a broad range of risk.

The true impact of a risk score depends on how it will be used to influence patient care and decision-making. In this effort, we devised a simple guide to categorize patients as low, average, above average, or high risk for renal failure after OHT, as shown in Table 3. This guide was based on data in the derivation cohort and confirmed to be accurate in the validation cohort. The range of risk is broad within the guide, with low-risk patients having less than 5\% risk and high-risk patients having greater than $20 \%$ risk of postoperative renal failure.

We believe this guide provides a broad enough range of renal failure risk to affect patient care. Moreover, decisions regarding fluid management and diuresis, use of nephrotoxic agents, including immunosuppressive agents and induction therapy, monitoring of renal function by conventional methods and newer technologies, and early institution of renal replacement therapy may be affected by such a guide. For example, a stronger case may be made to avoid aggressive diuresis and the use of nephrotoxic agents in the high-risk category. In addition, more frequent monitoring of renal function, including early consultation with nephrologists, may be more appropriate for higher-risk patients. The development of new urine or serum biomarkers for acute renal failure also may be higher yield and more cost-effective in this higher-risk category of recipients. $^{14}$

Another important application of the risk score and guide may be in translational and clinical research. For instance, induction therapy is currently used in approximately $50 \%$ of OHT recipients. ${ }^{15}$ Its main objectives are not only to provide early, intense immunosuppression but also to delay initiation of potentially nephrotoxic immunosuppressives beyond the early postoperative period when susceptibility to renal injury is greatest. Stratifying patients according to renal failure risk in clinical studies to better define the role of induction therapy in OHT may be helpful. In particular, patients in the highest risk category may benefit the most from avoidance of nephrotoxic agents. Furthermore, as previously mentioned, novel serum and urinary biomarkers are being developed for acute renal failure. ${ }^{14}$ The predictive values of these biomarkers may be greatest in the higher-risk categories where the prevalence of kidney failure is higher. In addition, using these biomarkers to monitor for renal failure postoperatively 
may be most cost-effective in these higher-risk categories, again because the prevalence will be higher and costs associated with renal failure potentially avoided if clinical action can be undertaken to prevent further renal deterioration and eliminate the need for dialysis.

Finally, our composite risk score and guide may have utility in informing patients and their families about OHT and its associated risks. More specifically, the guide in Table 3 can be used to give a realistic impression of what the risks are of needing dialysis at least temporarily in the postoperative period. Although our score is a predictor of acute renal failure and not necessarily the need for chronic dialysis, the former is certainly a risk factor for the latter. In a future study, it may be important to evaluate the specific rates of chronic dialysis stratified by acute renal failure risk category, because ongoing dialysis would certainly affect a patient's quality of life and may therefore be important to disclose in the consenting process.

\section{Study Limitations}

An important limitation of our study is that other variables that may affect rates of renal failure are not available in the UNOS database and therefore are unable to be included in our risk index. These variables include the use of nephrotoxic medications, perioperative fluid balance, intraoperative and postoperative bleeding, and episodes of perioperative hypotension, to name a few. In addition, the decision to dialyze a patient can vary between providers and between institutions. There is a possibility that a patient who was categorized as having renal failure requiring dialysis in our study may not have been dialyzed with a different provider or at a different institution. Another limitation is that our analysis was confined to the postoperative period, but there is a possibility that an OHT recipient may have been readmitted after hospital discharge and subsequently dialyzed. Finally, our analysis is limited to renal failure requiring dialysis and did not evaluate less severe forms of acute kidney injury, because these data are not available in the UNOS registry.

\section{CONCLUSIONS}

This study derived and validated a composite risk index for postoperative acute renal failure requiring dialysis after OHT using data from 14,635 patients. The risk score was then used to construct a simple clinical guide to provide clinicians the ability to prospectively estimate postoperative renal failure risk in OHT recipients. The risk index and clinical guide may have potential applications in guiding the perioperative management of OHT recipients and in clinical research stratification. Because postoperative acute renal failure is a powerful predictor of mortality, other morbidity, and resource use, continued improvement in preventing its occurrence and optimizing its management is essential.

\section{References}

1. Escoresca Ortega AM, Ruiz de Azua Lopez Z, Hinojosa Perez R, Ferrandiz Millon CM, Diaz Martin A, Corcia Palomo Y, et al. Kidney failure after heart transplantation. Transplant Proc. 2010;42:3193-5.

2. De Santo LS, Romano G, Amarelli C, Maiello C, Baldascino F, Bancone C, et al. Implications of acute kidney injury after heart transplantation: what a surgeon should know. Eur J Cardiothorac Surg. 2011;40:1355-61.

3. Turker M, Zeyneloglu P, Sezgin A, Pirat A, Arslan G. RIFLE criteria for acute kidney dysfunction following heart transplantation: incidence and risk factors. Transplant Proc. 2013;45:3534-7.

4. Boyle JM, Moualla S, Arrigain S, Worley S, Bakri MH, Starling RC, et al. Risks and outcomes of acute kidney injury requiring dialysis after cardiac transplantation. Am J Kidney Dis. 2006;48:787-96.

5. Ojo AO, Held PJ, Port FK, Wolfe RA, Leichtman AB, Young EW, et al. Chronic renal failure after transplantation of a nonrenal organ. $N$ Engl J Med. 2003;349:931-40.

6. Dasta JF, Kane-Gill SL, Durtschi AJ, Pathak DS, Kellum JA. Costs and outcomes of acute kidney injury (AKI) following cardiac surgery. Nephrol Dial Transplant. 2008;23:1970-4.

7. Patel ND, Weiss ES, Allen JG, Russell SD, Shah AS, Vricella LA, et al. Heart transplantation for adults with congenital heart disease: analysis of the United Network for Organ Sharing database. Ann Thorac Surg. 2009;88:814-21.

8. Gueler F, Hanke N, Wiese B, Simon A, Haller H, Haverich A, et al. Acute kidney injury after heart and/or lung transplantation: retrospective analysis of incidence, risk factors and outcome of 1400 patients. Nephrol Dial Transplant. 2013; 28(Suppl 1):i73.

9. Rylski B, Berchtold-Herz M, Olschewski M, Zeh W, Schlensak C, Siepe M, et al. Reducing the ischemic time of donor hearts will decrease morbidity and costs of cardiac transplantations. Interact Cardiovasc Thorac Surg. 2010;10:945-7.

10. Blanche C, Nessim S, Quartel A, Takkenberg JJ, Aleksic I, Cohen M, et al. Heart transplantation with bicaval and pulmonary venous anastomoses. A hemodynamic analysis of the first 117 patients. J Cardiovasc Surg (Torino). 1997;38:561-6.

11. Aziz T, Burgess M, Khafagy R, Wynn Hann A, Campbell C, Rahman A, et al. Bicaval and standard techniques in orthotopic heart transplantation: mediumterm experience in cardiac performance and survival. J Thorac Cardiovasc Surg. 1999;118:115-22.

12. Shahian DM, O'Brien SM, Filardo G, Ferraris VA, Haan CK, Rich JB, et al. The Society of Thoracic Surgeons 2008 cardiac surgery risk models: part 1-coronary artery bypass grafting surgery. Ann Thorac Surg. 2009;88:S2-22.

13. O'Brien SM, Shahian DM, Filardo G, Ferraris VA, Haan CK, Rich JB, et al. The Society of Thoracic Surgeons 2008 cardiac surgery risk models: part 2-isolated valve surgery. Ann Thorac Surg. 2009;88:S23-42.

14. Cruz DN, Bagshaw SM, Maisel A, Lewington A, Thadhani R, Chakravarthi R, et al. Use of biomarkers to assess prognosis and guide management of patients with acute kidney injury. Contrib Nephrol. 2013;182:45-64.

15. Stehlik J, Edwards LB, Kucheryavaya AY, Benden C, Christie JD, Dipchand AI, et al. The Registry of the International Society for Heart and Lung Transplantation: 29th official adult heart transplant report-2012. J Heart Lung Transplant. 2012;31:1052-64.

\section{Discussion}

Dr Mark Slaughter (Louisville, Ky). In the preoperative variables that were evaluated, it appears as though transpulmonary gradient and pulmonary vascular resistance were not included. These can affect donor right ventricular function and lead to low-flow output situations and poor renal perfusion. Were these variables not available, and what influence would they have on the risk score if included?

Dr Kilic. Some hemodynamic variables are inconsistently recorded in the UNOS database. Those variables that had more than $20 \%$ missing data were excluded as potential risk factors in our score calculation. Other hemodynamic variables did not meet entry criteria for our multivariable model. The other item with the hemodynamic parameters is exactly when they're being measured. In the UNOS database, they are measured as close to 
the heart transplantation as possible, which I think is a reliable measure. However, again because of the high percentage of missing data or the lack of predictive power, those variables were excluded.

Dr Slaughter. Similarly, for preoperative variables, I'm surprised that status $1 \mathrm{~A}$ and $1 \mathrm{~B}$ were not included. You would think a higher-risk individual might be at higher risk for renal failure.

Dr Kilic. We looked at each component of what would determine a patient to be UNOS status 1 or 2 . So bridging with ventricular assist devices, extracorporeal membrane oxygenation, patients on inotropes, all those variables were looked at and evaluated in the analysis. Some of those variables were significant in univariate analysis; however, after our statistical methodology, they did not meet inclusion criteria for the final multivariable model.

Dr Slaughter. Although mentioned as limitations, operative events that result in bleeding, transfusion, hypotension, and decreased donor organ function will significantly affect postoperative renal function. Do you think your risk score is just a surrogate marker for those patients who will not tolerate perioperative complications?

Dr Kilic. That's an excellent point, and it is something we can't control for because those variables are not present in the UNOS database. So it's difficult to tell. It may very well be a surrogate.

Dr Slaughter. The concern would be if you have a patient with a predicted $20 \%$ risk. The question is, are you going to exclude that patient? But if you have a perfect operation, once again, the odds are the patient probably will be okay.

Last, along these lines, will the risk score change your approach to managing patients? An example is a patient with decreased renal function, diabetes, and age more than 60 years, thus a high-risk patient for post-transplant renal failure; should that patient automatically get a ventricular assist device to demonstrate improvement in renal function before receiving a transplant that might lead to better organ allocation?

Dr Kilic. That's one of the potential applications of this risk score. Other applications would be looking at induction therapy, for instance. Approximately $50 \%$ of transplant recipients receive induction therapy, and it would be interesting to look at those patients who are at very high risk for renal failure preoperatively whether or not induction therapy would be more useful or not. These are all potential clinical applications and certainly deserve more attention.

Dr Robert Higgins (Columbus, Ohio). Yes, I agree, Dr Kilic, a very articulate description of a complicated issue. I guess the question I had in this risk-adverse world, knowing that we are trying to optimize outcomes for these heart transplant recipients, is should we now use this tool as a discriminator of patients who are at risk for renal failure in the perioperative period? Can creatinine clearance less than 30 be a marker for impending renal failure postoperatively? Maybe they shouldn't even be considered for heart transplant alone but rather heart-kidney transplantation, which would then allow you to mitigate the effects of whatever risk factors or variables are being played out here.

Dr Kilic. When we're talking about patient selection, which patients should undergo heart transplantation, we should focus on models that are looking at postoperative or 1-year mortality risk. Renal failure is a strong predictor for mortality, but there are other components that certainly contribute to overall mortality risk.

The utility of this score is to specifically tailor certain aspects of postoperative management and in terms of clinical research stratification. In terms of being a score that we look at for determining patient selection, again, I think models that are aimed at measuring risk of overall mortality are the models we should look at for that.

Dr Higgins. Yes, this may be a tool that would be effective for a lot of people.

Dr Thoralf Sundt (Boston, Mass). My question relates to having sat for many hours in transplant selection conferences agonizing over whether the patient ought to have a heart or a heart-kidney. How many times have we had that discussion? The cardiologist or nephrologist says, "Oh, the kidneys will get better," and sometimes you say it too.

Are you able to look within your own institutional database at those patients who had dodgy renal function preoperatively and separate those who did or did not get better and use this score to distinguish them? Is this actually applicable to the related question, will somebody with preoperative renal failure get better after a transplant or not?

Dr Kilic. Yes. Again, unfortunately, the UNOS database is limited in terms of the amount of postoperative data it has. Renal failure is simply coded as whether it's present or not, and that's only in cases where dialysis is needed. But I agree, looking at a single institution series where you have more clinical data available, including postoperative creatinine levels and other markers of renal failure, would be very interesting.

Dr Sundt. That's where you'd need to do it, in your own database. Thanks.

Dr Glenn Whitman (Baltimore, $M d$ ). We too suffer every Tuesday afternoon with the dilemma of doing an isolated heart transplant versus a dual-organ transplant, pulling the kidney with the heart. Or, if we do not do a dual transplant, should we oversize because of worry about right ventricular failure, elevated central venous pressure, and postoperative renal function. But we can't do that study Dr Sundt just mentioned because we already use induction therapy selectively in patients who we think are going to have a high risk of renal insufficiency. Induction therapy is expensive, so we do not use it uniformly, but when someone is at high risk, we use it. 